\title{
The effect of scaling methods on the calculation of environmental indices
}

\author{
Joanna Adamczyk \\ Faculty of Forestry, Warsaw University of Life Sciences - SGGW \\ Nowoursynowska 159, 02-776 Warsaw, Poland \\ e-mail: joanna_adamczyk@sggw.pl
}

\begin{abstract}
Landscape structure quantification is a subject of great interest in the environmental sciences because of the practical advantages it offers, including calculation of the environmental indices useful for land management, ecology and many other fields. A trend of developing new systems of environmental indices can be observed in European Institutions such as EEA and EUROSTAT, but there has been criticism about approaches based on Corine Land Cover (CLC). One of the aims of this article is to review the method of this database preparation for the purpose of calculating environmental indices. This study tests the ability of three methods to scale categorical maps and retaining as much of the original landscape structure information as possible. The vector scaling method is comparable to the preparation of the CLC data. Two other methods use Geo-Object Image Analysis as the main tool for classification. The scaling is performed in this method through building a hierarchy of objects and scaling the raster imagery. The results are compared and evaluated for scale effects and the calculation of environmental indices on the basis of the scaled data. There is no universal method for all the characteristics of the landscape pattern. The GEOBIA-based methods demonstrate greater applicability to fine grained structural and landscape configuration analyses. The vector scaling method is applicable mainly to landscape configuration, its results are also better for visualization of the scaled map.
\end{abstract}

Key words: spatial scaling, remote sensing, GEOBIA, MAUP, generalization

\section{Introduction}

Landscape structure quantification is a subject of great interest in the environmental sciences because of the practical advantages it offers. The landscape structure indices used for this purpose are based on the widely adopted assumption that there is a possibility of describing the ecological value of the landscape on the basis of its structural assessment of, for example, biodiversity, intensity of landscape transformation, and population dynamics (McGarigal 2006). The results are useful for land management, ecology and many other fields. On the high level of environmental decisions the wide application of environmental indices for decision support is observed. European institutions such as the European Environment Agency (EEA) and EUROSTAT are currently working on the development of new, more detailed systems of environmental indices, such as EBONE (2011) or MCPFE (2011). This approach has the practical advantage of allowing us to quantify the characteristics of the environment, but some criticisms have been voiced regarding the methods applied for calculation of these indices with widely adopted datasets such as Corine Land Cover. Some authors, such as Solon (2004), argue that land cover is not the best way to characterize the ecological value of the landscape, and that much better results would be given by patches of actual vegetation, less transformed by interpretation. Other authors indicate that taking into account only vertical landscape structure is not sufficient for such assessments and suggest that landscapes can also be characterized according to the hierarchical dependencies between the levels of landscape organization (Allen \& Starr 1982). 
Scale and scaling are said by many authors (e.g. Levin 1992) to be the central issue in landscape ecology. There is a widely accepted view that the processes occur at different levels of scale and cannot be observed on only one of them (Marceau 1999). The processes are also subject to hierarchical relationships related to the scale of observation and their scaling is an integral part of understanding these processes (Levin 1992; Marceau 1999). Despite many years of research issues regarding the scaling of spatial information are still a challenge. Researchers are still trying to find a method for determining the 'optimal scale' for analysis (Marceau et al. 1994; Wu et al. 2000), and to treat the interpretation of landscape metrics in different scales and on different levels of generalization (Marceau 1999).

Scaling spatial information poses technical problems that can lead to increased uncertainty regarding data (Wu 2004). The process performed for categorical maps containing land cover cannot be compared to generalization of topographical maps. There are problems with such data: modifiable areal unit problem (MAUP); nonlinearity of dependencies between the phenomena; and processes across the hierarchy of scales. The landscape heterogeneity is mentioned as one of the more complicating the process of scaling (Wu et al. 2000).

There are many frameworks for scaling categorical maps, but Geo-Object Based Image Analysis (GEOBIA) is widely tecommanded preferred for reducing the technical problems (Marceau \& Hay 1999). The advantages of this method are related to its different approach to image classification, based on the similarity of the approach to human interpretation of remote sensing imagery and explicit building of object hierarchy. Given the high potential of this method three scaling frameworks were developed that were based on the direct object-based approach:

- Scale-Space Blob Feature detection (Lindeberg 1994; Hay et al. 2002) - offering 3D space (blob) of scaled objects at which the disappearance and emergence of the objects can be observed. It is based on Gaussian filtering of the source images;

- Fractal Net Evolution Approach (FNEA) (Baatz \& Schaepe 2000) - implemented in the eCognition software method of building a hierarchy of objects based on the segmentation and scaling parameters. This approach was used in this study, because of its explicit building of the scale hierarchy;

- Multiscale Objects Specific Analysis (MOSA) (Hay 2002) - building of the object hierarchy is preceded by Object Specific Analysis, a part of these method Estimation of Scale Parameter (Drăguţ et al. 2010) was used in the present study. The scaling approach is based on the deeper analysis of the image properties in comparison with the FNEA.

There are at least two reasons for scaling spatial information: performing spatial analysis on a different scale from the data acquisition scale and the necessity to have the information generalized in space and time but based on particular research, e.g. at the ecosystem level. Calculation of the ecological indices is also an example of the second application.

The purpose of this study was to analyse how information about the landscape structure at the basic class level is retained through the hierarchy of scales, using the following methods:

- Scaling of vector data is often performed when the preparation of the database from existing data is needed. For example, the first editions of Corine Land Cover were prepared from existing data in some countries such as Great Britain (Brown et al. 2002). The generalization of the categorical layer of land cover has included methodological aspects of geometrical and semantic generalization of two different data models;

- Scaling through aggregation of base raster data is also a popular method of scaling and is mainly used during integration of databases, when raster data are too fine for some applications, e.g. for a better view of certain spatial structures. It has been proved (Heuvelink 1992; Hunter \& Goodchild 1997) that statistics performed on aggregated data can improve the results of analyses when they are performed on a specific level of generalization. This approach is called indirect in the literature and was used in this study according to Marceau et al. (1994) as a mixed approach combining aggregation of raster images and classification at the generalization level; - The GEOBIA hierarchical scaling method is often regarded as the best, multipurpose method for scaling spatial data. The above-mentioned advantages determined the use of the FNEA approach for this study. An important reason for this decision was the easier comparability of this method with the other methods tested during this study than that of the results from MOSA or SS.

The main aim of the research is to compare the three methods in terms of the scale effects influencing calculation of environmental indices. The study is performed only on one scale range between two thresholds: the lower scale, which offers the possibility to delineate the basic land cover classes (e.g. kinds of crops); and the higher scale, in which the classification schema does not have to be aggregated to the types of land cover (e.g. arable land). The tests are focused on retaining the important characteristics of the land cover structure through the scale hierarchy.

\section{Study area and material}

The case study was carried out in the eastern part of the Warsaw Metropolitan Area in Poland. The study area of ap- 
prox. $3600 \mathrm{~km}^{2}$ is covered by one scene from the SPOT5 satellite. The 4 band orthorectified product was used, at a spatial resolution resampled to $10 \mathrm{~m}$. The scene centre is $52^{\circ} .2022^{\circ} .80$ (WGS 84). The covered area represents a complex landscape structure with patches of complicated shape and of different size: forest patches in some parts highly fragmented, in other parts with high connectivity.

The acquisition date of 17 July 2006 allowed the full spectrum of available land cover types for this area to be represented. The class hierarchy of the object-based classification made possible to distinguish: coniferous and deciduous forests, pasture and meadows, four types of arable land, identified from reference data as crops, bare ground, and fallow land with elements of natural vegetation, roots and brassicas. The reference material used for examining accuracy of the base classification was acquired from the master map and the in-field documentation from which 297 reliable test sites were selected as the reference for assessment of classification accuracy.

\section{Methods}

The present study is not focused on development of the workflow for scaling categorical maps. The aim was to analyse one part of the scaling process. It is one scale range between the original image scale, where the information is obtained from the satellite image and processed to the categorical map, and the highest level of the scaled-up map before the scale threshold, when the attributes of land cover classification are generalized.

The tested issue related to retaining important information about the landscape structure from the results of classification on the up-scaled levels. The tests were performed on a remote sensing image with a pixel dimension of $10 \mathrm{~m}$. The classification of the image was done with Geo-Object Based Image Analysis (GEOBIA), and the results were taken as a basis for comparison of the scaling procedures and the effects on landscape pattern analysis. Three methods of spatial data scaling were tested:

- GEOBIA hierarchical scaling - performed on the basis of remote sensing images, incorporating the building of an object hierarchy.

- GEOBIA scaling of raster images, across levels of increasing spatial resolution of raster images.

- Vector layer generalization using standard method, implemented in the commonly used GIS software.

The base layer (raster or vector) was up-scaled over a range of spatial scales, and the land cover classification was derived from each of them. Quantitative characteristics of land cover were calculated for each scale level in order to evaluate ability to represent the spatial pattern. The most important characteristics allowing complete de- scription of the landscape pattern from the ecological perspective can be classified in the following groups:

- comparativeness and membership of the up-scaled objects between the levels of scale;

- the inside patch homogeneity - allowing assessment of the representation of the real-world land cover;

- patch shape and form metrics;

- diversity of the landscape land cover (the measure was selected for comparison, but on the assumption that much better results are obtained when the diversity is calculated for the vegetation species patches).

The following assumptions were made in the research:

- the only changes applied during the scaling process were related to generalization of the geometric properties of the data, and the thematic attributes were not changed;

- the GEOBIA base classification and the results of hierarchical scaling were the basis for comparisons between the methods because of the acknowledged high accuracy of results;

- the base scale level for the whole method was assessed for the GEOBIA hierarchical scaling and the other methods were tested with the same scale thresholds;

- the results of the preformed classifications would be as accurate as possible (the minimum desired Kappa Index was set at 0.90 );

- the uncertainty associated with the classification would be always taken into account during interpretation of the results;

- the classification results would be comparable for each level of scale.

The main challenges of the research are related to the comparability of the results and the scale level between the tested methods.

\subsection{Base classification}

The base classification was performed with Object Based Image Analysis (GEOBIA). The image set included four channels obtained from SPOT5 Satellite: B1 (green); B2 (red); B3 (near infrared); B4 (SWIR). The classification procedure was performed with the supervised Nearest Neighbor classification workflow implemented in the Trimble eCognition Developer software, in the following steps: multi-resolution segmentation, selection of sample objects, finding the best configuration of features, classification, accuracy classification assessment.

The multi-resolution segmentation was performed by means of parameters chosen by visual assessment testing whether the resulting objects properly represented land cover types distinguishable by the human eye. Special emphasis was placed on contrasting land cover patches of a forest type (including small groups of trees - environmental use areas), and arable land or meadows. The empir- 
ically chosen parameters for the segmentation were: scale 50 , shape 0.1 , compactness 0.4 .

The dataset containing the test areas was divided into two parts. The first 80 objects, proportionally representing the probability of occurrence of the types of classes in the image, constituted the sample dataset for classification. The other 117 sites were used for testing classification accuracy.

The defined classes of land cover were characterized by features, the typical characteristics of the objects belonging to each class. The best combinations of Nearest Neighbor features were calculated with the Feature Space Optimization tool, ${ }^{1}$ individually for two groups of classes: agricultural land and other land cover.

The classification accuracy was assessed with the Kappa Index of Agreement through comparison of the classification layer with the 117 test sites. The best accuracy achieved was 0.947 . The image, because of the high number of small complicated patches, was difficult to classify. The stability of classification ${ }^{2}$ was assessed as sufficient for further study.

\subsection{Hierarchical GEOBIA scaling}

The hierarchical scaling by Geo-Object Based Image Analysis was performed with the Fractal Net Evolution Approach (FNEA) (Baatz \& Schaepe 2000), implemented in eCognition software. The base classification performed on the original image was developed to the image object hierarchy. The new levels of image objects were generated above the base level, by increasing the level of scale (or in other words increasing the level of allowed objects heterogeneity).

The behaviour of image objects during the up-scaling segmentation was additionally assessed with the method introduced by Drăguţ and colleagues (2010) for estimating the appropriate scales for image segmentation ESP (Estimation Scale Parameter), available for Definiens Developer software. The objective was to predict the scale thresholds and the levels on which the best quality could be obtained. The tool performs segmentation across defined levels of scale and calculates the local variance parameter of heterogeneity for the objects in the whole scene. The results of the calculation are presented in a graph showing the rates of change of the variance parameter (Fig. 1). The local maxima of the parameter, according to Drăgut and

${ }^{1}$ Feature Space Optimisation (FSO) is a tool implemented in eCognition software. It is used in the Nearest Neighbor classification. The multidimensional mathematical method is used for calculation of the best separation distance between features applied to samples of classes.

2 The classification stability is calculated in eCognition software to appraise the quality of classification results. For this purpose the distance between two assignments of class (the best and the second best) is calculated: the shorter the distance, the less stable the classification. colleagues, are to be interpreted as the scale on which the homogeneity of the objects increases. This may be interpreted as the threshold of scale for the particular image on which the new level of meaningful image objects emerges. Performing the segmentation on the other levels of scale will result in only minor modifications to the structure of the objects.

The results (Fig. 1), compared with visual interpretation of the segmentation results, proved that the first scale parameter for segmentation should be 50 . The other levels were tested beforehand using an interval of 10 scale parameters. The graph interpretation suggests that the best results should be obtained for levels of 60, 70, 100, 120, and 150 . A lower quality of segmentation (and classification results) will be achieved for levels 70, 90, and 110 and those above 150. The visual comparison proved that the visible local maximum in scale 150 is the threshold for segmentation, allowing us to obtain the predefined patch types without generalization of the thematic attributes.

Based on the results from the ESP methods the parameters for development of the object hierarchy were determined. The test proved that the scaling should be performed using a 10-unit interval. As the starting parameter for base classification the level of 50 was selected. Each of the levels was classified using the same class hierarchy. The sample objects were also adopted from the base level, but some manual corrections were needed. The changes to the image object size caused the loss of some small samples. They were replaced manually in order to maintain the predefined proportions.

Each of the image object levels was classified twice: first, based on the same set of features describing the image classes and, second, by optimizing the set of features before each classification using Feature Space Optimization. The observed changes of feature space were not revolutionary, but demonstrated the important trends of changes. The results of accuracy assessment between levels of scale for the two methods proved that the second method, although much more laborious, gave more stable results. The optimization of the classification was performed as long as the desired level of accuracy (minimum KIA 0.90) was reached. The whole procedure or hierarchical classification was performed until visual assessment of the resulting classification suggested that the next level of scale was reached (e.g. types of crops were merged into the arable land, types of forests aggregated).

\subsection{Scaling by raster aggregation}

Scaling of the land cover layer through raster aggregation was performed in the following steps:

- choosing the raster aggregation method suitable for the requirements of this study and for retaining the land cover properties; 


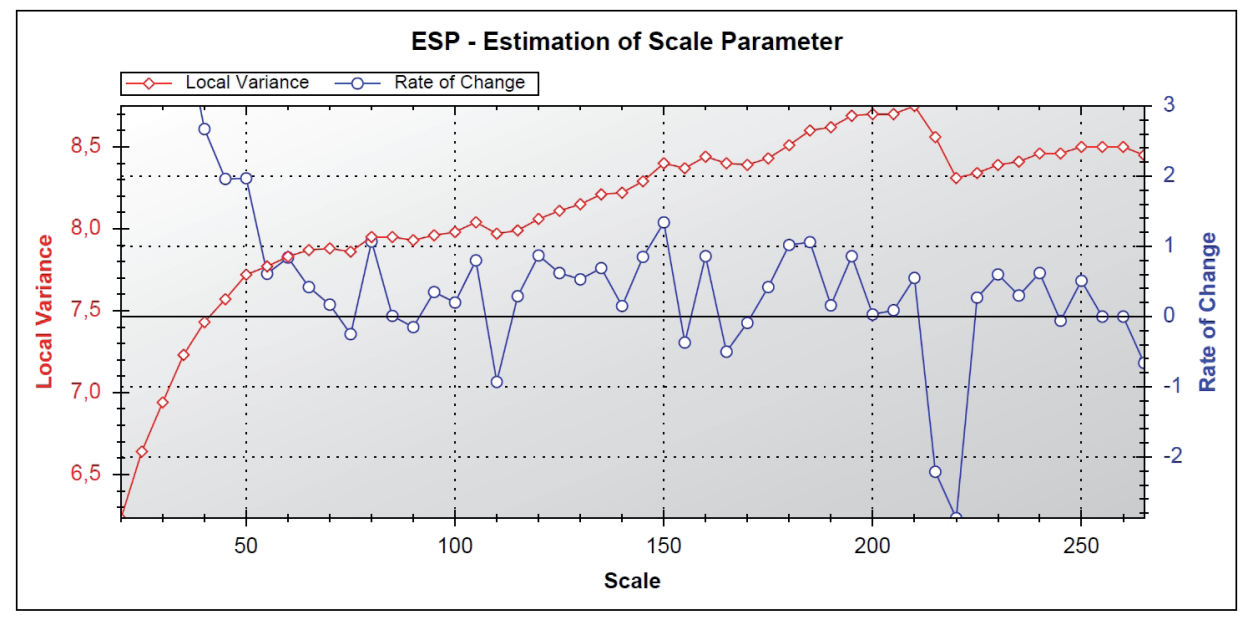

Figure 1. Results of the estimation of the scale parameter for the Warsaw Metropolitan Area on the SPOT5 image, performed with the increasing scale parameters of GEOBIA segmentation

- raster aggregation to the successive levels of resolution using the selected method;

- GEOBIA classification of the scaled images, based on a comparable rule set;

- checking of the classification accuracy.

The four resampling methods applied for raster aggregation were tested (Nearest Neighbor, Majority, Bilinear and Cubic) in order to identify the best one. The Nearest Neighbor (NN) method is recommended for categorical maps, since it does not change the value of the cells. The $\mathrm{NN}$ assignment was therefore performed, so the maximum spatial error was half of the cell size. The Majority (MJR) algorithm determines the new value of cells based on the most popular values within the filter window. It is also used for discrete data. The result is smoother than when the NN method is used, because the algorithm is based on an averaging of the values. The Bilinear Interpolation (BIL) method calculates a weighted distance average of four nearest input cell centres, and as a result a smoothing effect is observed. The algorithm is used for continuous data. The Cubic (CUB) method performs a cubic convolution determining the new cell value based on fitting a smooth curve through the 16 nearest input cells. It is used for continuous data, because is based on interpolation of the cell values. The results may contain cells of values outside the range of the input raster but are less geometrically distorted than the raster resulting from the $\mathrm{NN}$ algorithm.

The methods were tested for similarity of the two rasters after aggregation and optimal stability of the properties before and after scaling. This was done by measuring the RMS error of pixels' digital number between the source and scaled raster data. The test was performed on whole satellite scenes on certain scales in comparison with the base image of $10 \mathrm{~m}$ resolution. As presented in Figure 2a the maximum value of errors was obtained with the $\mathrm{NN}$ algorithm; the smallest difference between the cell values of the largest scale image was offered by the MJR method. The results from the other methods proved the above theory regarding the standard changes of cell values. The second smallest change of cell values, and stability for all three images, was offered by the BIL method.

The influence of the other characteristics of the scaling algorithms is presented in Figure $2 b$ where the variance of the digital number of pixels is assumed in comparison with the original image. The influence of the scaling method is again similar for the bilinear and cubic convolution algorithms. The visibly highest variance was caused by the NN method. The majority algorithm reduced the variance much more than other methods. The lowest change of the pixel values was offered by the majority and bilinear methods. The retaining of as much information as possible is related to satisfying the of high variance of the results, which is kept by only one of the methods - bilinear interpolation. This method was chosen for further research. The raster images were scaled at the interval of $10 \mathrm{~m}$ of spatial resolution.

The scaled images were classified with the NN supervised classification implemented in the GEOBIA software. The comparability of results was one of the main areas of concern. The segmentation parameters of colour, shape, smoothness, and compactness were the same as for the hierarchical GEOBIA method. But the scale parameter determination poses problems because there is no quantitative method to relate it to the raster resolution. This was tested empirically and the results are presented in section 3.5. Based on these results, the scale parameters for each 


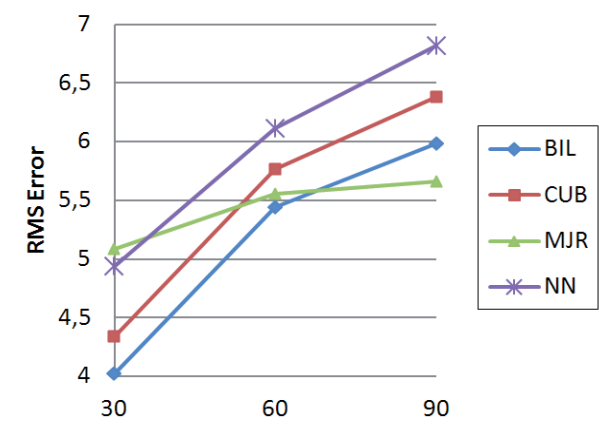

Figure 2. The RMS error (a) and variance (b) of digital number of pixels in images scaled using four tested methods: BIL - Bilinear Interpolation, CUB - Cubic Convolution, MJR - Majority Filtering, NN - Nearest Neighbor scaled raster resolution were adopted. The classification process was performed with the same sample objects as in the hierarchical GEOBIA, and the methodology for supplementing the class characteristics was also the same. The desired quality level for each classification was also above 0.9 of KIA.

\subsection{Vector model}

A vector model of the categorical map in this study was included to investigate how the Corine Land Cover database is prepared. The subject is becoming more and more important since the standard European environmental metrics are based on the CLC data. Thus, out of the range of available scaling approaches and algorithms, the most comparable method to that adopted by the European Environment Agency for Corine Land Cover preparation process was chosen for this study.

According to the technical guidelines used in the Corine LC project (Bossard et al. 2000; EEA 2007) and based on the national experience of the UK, Sweden and Finland, the data conversion from other sources, was done through a semi-automatic process in three stages:

- computerized processing - where the area of the base unit adopted was $>25$ ha, the smaller changes $<23$ ha were automatically amalgamated, and the patches in between left to "intelligent" exaggeration. Generalization of small changes was done automatically if the generalization situation was clear (membership of the semantically nearest class could be achieved);

- processing done by operator: handling polygons between 23 and 25 ha; generalization of small polygons, in case of difficulty;
- the dimensions of the minimum recognized area were adapted according to actual scale level and the standards used for this study.

The study required preparation of more than one layer on a certain scale. It caused modifications to some elements of the cited methodology. The following operations were not applied to the dataset:

- generalization, typification, or aggregation of the class schema, the classes were not prioritized;

- collapsing the polygon features to lines or points, usually done for better representation of the generalized features;

- other graphical operations, e.g. exaggeration of important features or refinement of visual properties.

The generalizations applied to geometry of patches were:

- aggregation - done by merging a group of individual small features in close proximity; the group was represented as one continuous area;

- amalgamation - joining the contiguous features, either by merging the area to the semantically closest one, or by splitting the area between the neighboring features;

- smoothing - relocation or shifting of a boundary, in order to remove the step-effect of the original raster cells, and to capture the significant trends of boundaries;

- simplification - reduction of boundary line complexity by removing changes of direction smaller than a certain threshold.

The workflow was as follows:

1. Removing adjacent polygons of the same classes from original object-based classification.

2. Scaling data to the successive levels of scale:

- agregating polygons according to the above given standard with parameters: 
- Aggregation distance between the polygon borders to be aggregated was empirically determined as $2 *$ the minimum detectable object dimension for the level of scale.

- Minimum area of polygon and hole to be retained were determined according to the minimum mapping area for the level of scale.

- Simplifying aggregated polygons - for this task the Wang algorithm (Wang \& Müller 1998) (also called Bend Simplify) was chosen, a decision based on its visually assessed better results in comparison with other widely used methods, e.g. Douglas-Peucker algorithm. In this method (Wang \& Müller 1998), the line under the procedure of scaling is composed of a series of bends (positive or negative), which are eliminated according to the defined base line (compared with the diameter of a circle). The parameters of simplification tolerance were defined according to Kozioł (2012), for the given pixel resolutions (Tab. 1). The minimum area was defined as the minimum detectable area size for each scale according to the same methodology.

3. Handling the uncertainties of resulting polygons

- amalgamating the overlapping polygons, according to the above defined standards;

- checking the adjacency of the lines, and aligning the non-adjacent lines with the main shape of source polygons.

\section{Comparability of the classification results}

Investigating the comparability of the scaling results was critical for the reliability of the results of this study. The all-important question was how to compare the classified images at a standardized level of scale.

One of the challenges of this research was achieving comparability of the results between the GEOBIA classification based on homogeneity parameter and the resolution or scale of other data. Methods for quantitative comparison have not yet been developed, assuming it would be possible to do it. Therefore the comparison had to be based on other solutions known from the literature.

The analysis started with finding a relation between the raster resolution, map scale and minimum detectable terrain object size. The relation is treated in the literature in two ways. The more frequent method of calculating it is as follows: image resolution $=$ minimum detectable size . Such an approach is found in many sources, and also in many methodological guidelines and instructions. There are some reliable theories, however (e.g. Tobler 1988; Kurczyński 2006), which suggest that this method is incorrect. The relation between remote sensing image resolution was defined on the basis of the sampling theorem by Kurczyński (2006) as:
2GroundPixelSize $\leq$ Ground $R$ solvedDis tan $\boldsymbol{e} \leq 2 \sqrt{2}$ GroundPixelSize

The relation between parameters of scale and resolution was defined by Tobler (1988). He used the following equation: Map Scale $=$ Raster Resolution (in metres) $* 2$ * 1000. For example, for satellite imagery at a spatial resolution of 30 metres the detectable sizes are: linear object $30 \mathrm{~m}$ and area $900 \mathrm{~m}^{2}$. The mapping scale for this resolution is 1:60 000 or smaller. The last parameter is given only for comparison of the level of detail. It cannot be used directly as a reference, because the relation between image resolution and map scale depends on a range of conditions regarding inter alia the quality of the images and the desired map requirements (Kurczyński 2006) and is not defined by mathematical relationships. The relation between ground pixel size and ground resolved distance adopted for this study is given in Table 1. The parameters for scaling the vector layers, as mentioned earlier, were determined in accordance with Kozioł (2012) parameters of the simplification tolerance for the defined levels of scale (Tab. 1).

Table 1. Relations between base raster resolution, detectable object size (Kurczyński 2006), map scale (Tobler 1988), and simplification tolerance for the Wang simplification algorithm (Kozioł 2012)

\begin{tabular}{|c|c|c|c|c|}
\hline $\begin{array}{l}\text { Raster } \\
\text { resolution } \\
\text { (metres) }\end{array}$ & $\begin{array}{l}\text { Ground } \\
\text { resolved } \\
\text { distance } \\
\text { (metres) }\end{array}$ & $\begin{array}{l}\text { Minimum } \\
\text { mapping } \\
\text { area (square } \\
\text { metres) }\end{array}$ & Map scale & $\begin{array}{c}\text { Simplification } \\
\text { tolerance } \\
\text { (metres) }\end{array}$ \\
\hline 5 & 10 & 100 & $1: 10000$ & 3.325 \\
\hline 10 & 20 & 400 & $1: 20000$ & 6.65 \\
\hline 50 & 100 & 10000 & $1: 100000$ & 33.245 \\
\hline 100 & 200 & 40000 & $1: 200000$ & 66.49 \\
\hline 150 & 300 & 90000 & $1: 300000$ & 83.113 \\
\hline
\end{tabular}

The other issue for resolution was to identify the scale of the classification results from the GEOBIA, as the scale parameter cannot be directly compared with the map scale or raster resolution. The following example explains the difference between the two issues: GEOBIA is based on segmentation of the image into a set of discrete parts, each of them can be recognized as meaningful. The parameters for segmentation are based on the scale parameter, and determine the maximum level of heterogeneity allowed for the objects. In the image of the more complex structure, the smaller objects are segmented with the same scale parameter. If segmenting of objects of comparable size were the main task, the objects would have different meaning in different images, e.g. segmentation of more homogeneous image would result in delineation of individual fields of 

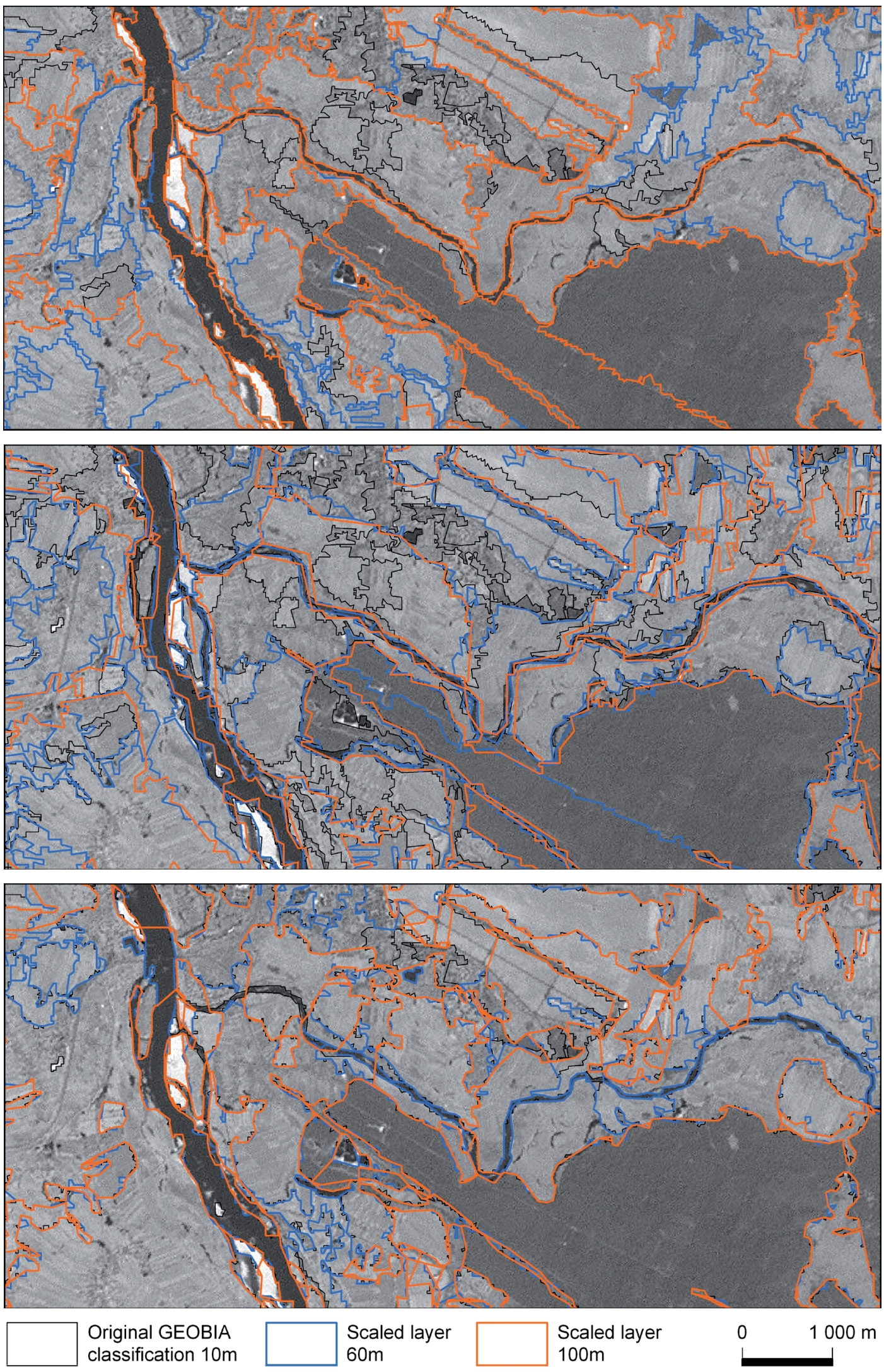

Figure 3. Comparison of the segmentation results from the three methods for two sample scales: a) hierarchical GEOBIA, b) GEOBIA raster, c) vector generalization 
different types of crops. Segmented objects of the same size in more heterogeneous images might delineate fields of crops, with mixed crops inside. The scale parameter would have to be much larger to achieve this. The two maps would be not comparable in any respect.

A comparison was performed for selected levels of scale, theoretically offering the best result of segmentation (based on the results of ESP analysis in Figure 1). The segmented layers on the chosen levels $(60,70,100$, $120,150)$ of results were compared by visual assessment with the whole range of the segmentation results from the GEOBIA raster and vector generalizations. The similarity was based on the most similar size and content of the segmented objects and the similarity of the object borders. The results are presented for selected examples in Figure 3.

The corresponding scale parameters and raster resolutions are presented in Table 2.

Table 2. Comparison between the scale parameters and raster resolution for two methods of scaling using GEOBIA, hierarchical and raster, for the satellite image of Warsaw Metropolitan Area.

\begin{tabular}{|c|c|c|}
\hline $\begin{array}{c}\text { Hierarchical GEOBIA } \\
\text { scale parameter }\end{array}$ & $\begin{array}{c}\text { Raster } \\
\text { resolution }\end{array}$ & $\begin{array}{c}\text { Scale parameter } \\
\text { GEOBIA raster }\end{array}$ \\
\hline 50 & 10 & 50 \\
\hline 70 & 30 & 45 \\
\hline 130 & 90 & 25 \\
\hline
\end{tabular}

It is important to stress that the given results are not universal for each image even when the spatial and spectral resolutions are same. The appearance of the resulting objects is strongly dependent on the structure of the land cover determining the segmentation parameters. Thus such assessment has to be performed whenever the new research is performed. The given relations between grain and scale parameter of the scaled raster image should also not be taken as standard.

\section{Results}

There are two important issues to be explored by comparison of the scaled data when characterization of the landscape pattern is the main goal of data preparation: the similarity of the objects between the scaled levels should be as high as possible; and the key properties of the resulting layers should offer the possibility to calculate the informative indices at the higher levels of generalization. The other standard property of the dataset which is expected after the scaling procedure is the usefulness for visualization. The research question was whether these benefits could be attained, and if so with which method and under what condition.

\subsection{Comparison of classification results}

To explore the accuracy of the representation of the real-world objects the methodology developed by Drăguţ and colleagues (2010) was adopted, as mentioned above. The assumption was that the local peaks of the objects resulting from the classification corresponded to the most meaningful objects. For purposes of comparison between the scaling results the mean homogeneity of the objects at each scaling level was calculated with the Gray Level Concurrence Matrix (GLCM) Homogeneity Measure by Haralick (1979).

The GLCM is a tabulation of how often different combinations of pixel grey levels occur in a scene. The parameter was calculated for pixels enclosed in the image objects for all the analysed layers of images. The value of the matrix was the normalized number of pair occurrences (number of occurrences number of all pairs).

The value of homogeneity was calculated inside the GLCM with the following equation:

$$
\text { GLCMHomogeneity }=\sum_{i . j=0}^{N-1} \frac{P_{i j}}{1+(i-j)^{2}}
$$

where:

$\mathrm{i}$ - the row number

$\mathrm{j}$ - the column number

$P_{i, j}$ - the normalized value in the cell $i ; j$

$\mathrm{N}$ - the number of rows or columns

The index values were within the range $[0 ; 1]$. The algorithm explores if the values are locally homogeneous by weighting the values by the inverse of the contrast weight with weights, the result decreases exponentially according to their distance to the diagonal.

The results were normalized by calculating the Rate of Change (ROC), the measure used in stock market analyses to explore the change in e.g. prices over a period of time. The ROC is calculated with the following equation:

$R O C=\left[\frac{H-(H-1)}{H-1}\right]$

where $\mathrm{H}$ is the GLCM homogeneity, on the current level, and $\mathrm{H}-1$ is the GLCM homogeneity on the level below. The normalization allows us to explore the homogeneity of image objects without increasing the grain of analysis. Then each of the rates was normalized by the original (not scaled) image to avoid the cumulative effect of the changes. 

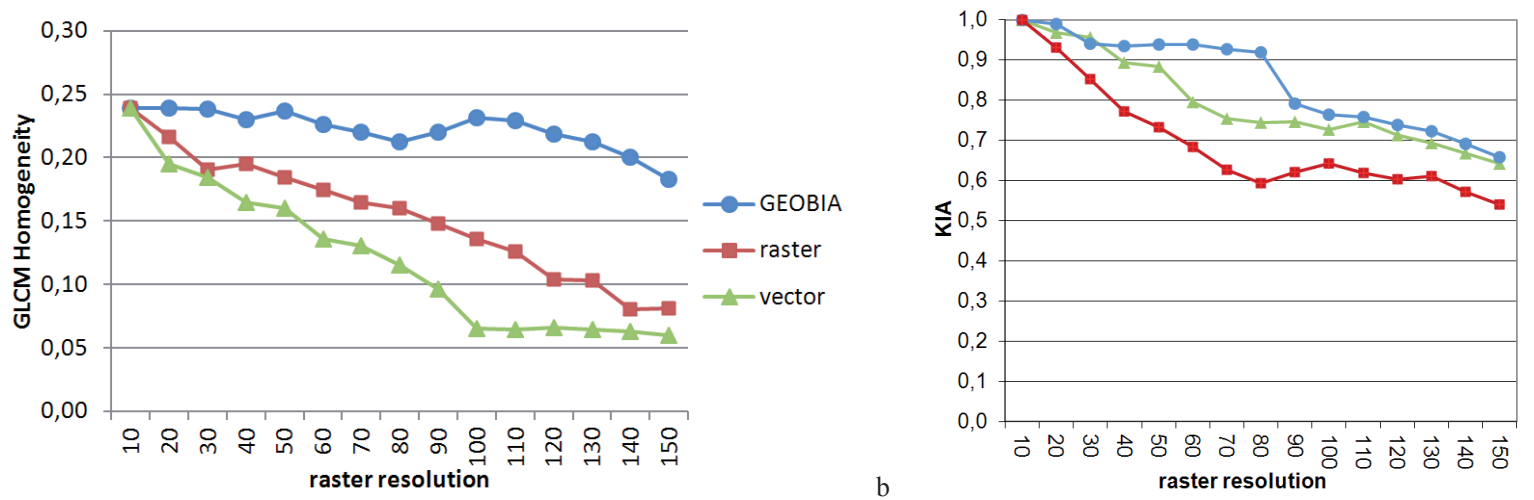

Figure 4. Rate of change of the mean GLCM homogeneity (a) and the Kappa Index of Agreement (KIA) (b) between the layers scaled by three methods: hierarchical GEOBIA, GEOBIA raster and vector scaling

The results (Fig. 4a) suggest the ability of the three methods to represent the real-world patches. The hierarchical GEOBIA adds only a small factor to the natural changes in image homogeneity caused by the application of the scaling procedure. The GEOBIA raster method lowers the parameter because of its greater ability to merge the objects; the greater decrease in the objects' homogeneity is caused by the vector scaling method. The reason is probably related to the significant changes in the geometry of the object borders.

The issue of changes to the whole image introduced by the three scaling methods was explored by assessment of the agreement of the classification results between all the scaled layers and the base classification. The comparison was performed under the assumption that the base classification layer (of known error compared with field measurement data) represented the truth on the ground. Such approximation allowed us to test the scaled layers by using more samples than the number of test areas available from the field data. For this purpose a set of 4000 points was generated, scattered randomly over the whole image.

The statistics of the attributed classes for each image were calculated with the Kappa Index of Agreement, a standard tool for classification quality assessment. It is stressed that the results presented in Figure $4 \mathrm{~b}$ do not represent the quality of the classification (as usually meant). The intention of this study was to compare agreement between the scaled images and the base layer, in order to assess the change between the layers influenced by the scaling method. What can be interpreted here is the ability of the methods to preserve the original content of the base classification after scaling.

The changes in the agreement are impacted by the classification method. As mentioned above, the classifications performed at each level were of the best possible quality. Nevertheless the results should be interpreted with caution as the errors introduced by classification should be taken into account.

The results demonstrate the specific changes in the classified images, which were dependent on the applied methods. The hierarchical GEOBIA eliminates small areas first, and after the threshold with a homogeneity of 110 is reached the large objects are merged and the classification agreement is reduced. The GEOBIA raster method merges objects at a much earlier level. The results are also less stable between the levels of scale. Vector generalization preserves the high level of similarity between the layers, because only small objects are eliminated, depending on the area. Also, the shapes of polygons are simplified, but the overall rate of change is smaller, because the large polygons are not merged. The higher levels have similar results to the hierarchical GEOBIA method, which is caused by changes in object borders which are very different at the higher scale levels.

\subsection{Ability to characterize the landscape structure}

The comparison of the key characteristics of the landscape structure was performed to take into account the applicability of the results for preparation of the material for calculating the environmental indices reflecting original properties of the landscape under analysis:

- heterogeneity of patches and the proportions between the patches of different types. The need to retain this characteristic raises the question of how long is the range of the scales with the same class hierarchy.

- retaining small areas of high importance, with simultaneous aggregation of other similar objects;

- shape of objects; special emphasis was placed on the complexity of shape, an important environmental indicator. 

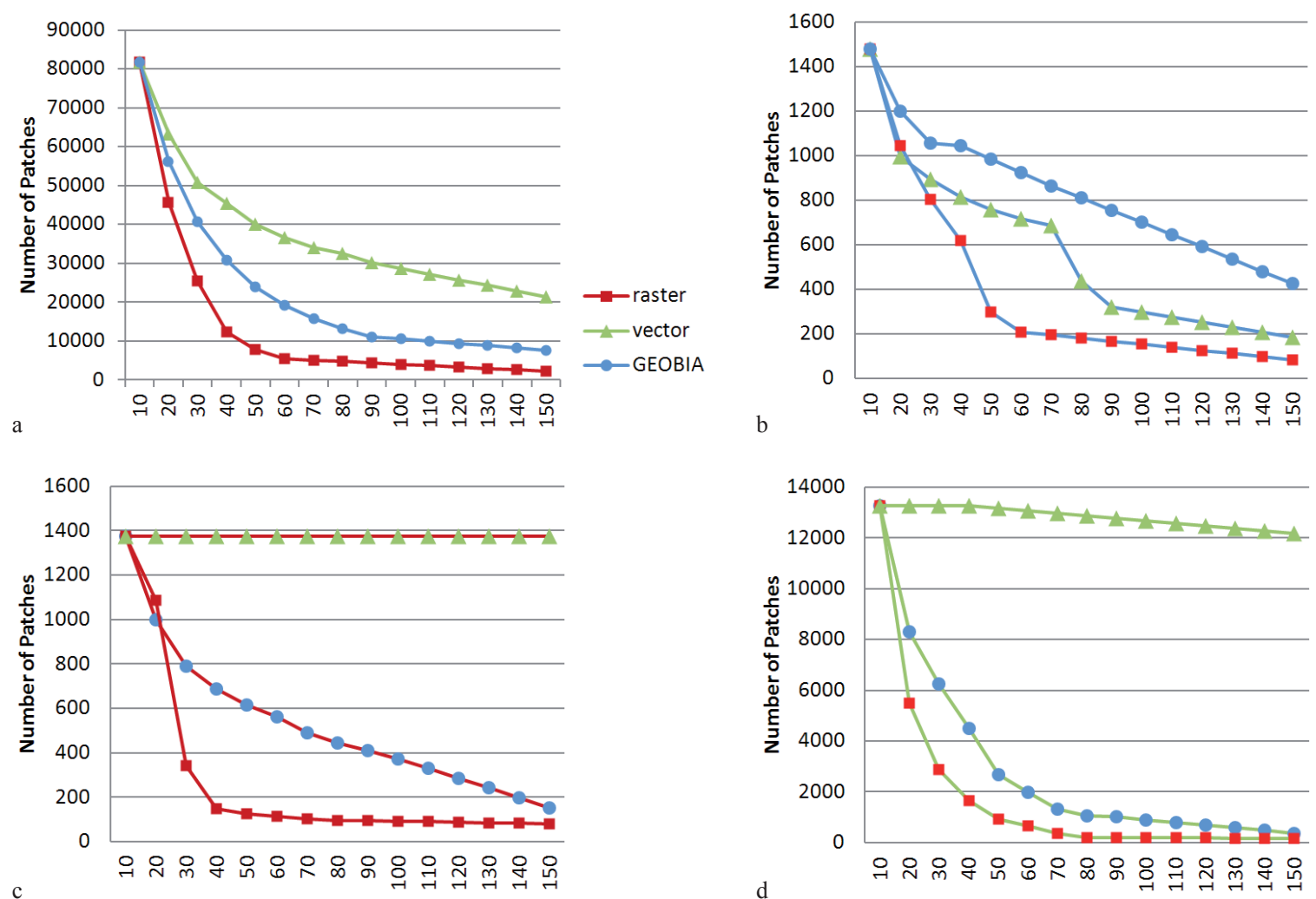

Figure 5. Number of patches in the layers scaled with three methods: GEOBIA hierarchical scaling, GEOBIA raster and vector scaling: a) all patches, b) small objects (water), c) medium objects (meadows), d) large objects (coniferous forests)

\section{Number of patches}

The number of patches on the levels of scale is compared in Figure 5a for the whole image, and in Figures 5b,c,d for the objects of different sizes. The results are comparable to the diversity metrics (Shannon-Weaver and Shannon Evenness) also calculated for the analysed layers. The results of NP are shown because the index is currently one of those most frequently used for diversity assessment in the European sets of indicators (e.g. SEBI 2012).

For all patches the comparison shows the similarity of the results for the GEOBIA methods and the vector aggregation in the overall shape. The in the particular results, however, the important effects are visible of the three different methods of scaling. Vector generalization preserves more objects than other methods because only the smallest objects are aggregated at the beginning of the scaling procedure. The overall number of patches preserves the diversity. The GEOBIA hierarchical scaling is stable for merging objects because it is independent of the properties of the shapes themselves. The basis for object aggregation is provided by the image context of the objects, the level of heterogeneity considered and the similarity between classes. GEOBIA raster scaling leads to dissolution of the small patches so the number of patches decreases rapidly. After a level of generalization of about 50-60 m of raster resolution, the more distinguishable patches are preserved, and the overall results are more stable. Patches of all sizes seem to be eliminated in a similar way.

The comparison of the patches of specific sizes was performed on selected classes representing the specific class area range: small $<200000 \mathrm{~m}^{2}$, medium -250000 $350000 \mathrm{~m}^{2}$, large $>400000 \mathrm{~m}^{2}$. The sample classes were selected according to their distinguishability in the neighbourhood.

The water class was selected (Fig. 5b), as the example of small patches, since removing it from the land cover map is not always desirable. For example the small isolated patches of forest and water should preserved when similar patches of arable lands are merged. The small patches are eliminated from the vector layer in a stable way and much faster than in the GEOBIA method, where the elimination of patches depends on their heterogeneity and many of them are preserved. In vector aggregation the threshold in elimination of the small patches is dependent on the number of objects of the specific size (area); also, the small 
objects close to the larger patches of the same type are merged. In the GEOBIA raster some of the smallest areas are averaged during raster aggregation, especially when they are similar to the neighbourhood.

The number of medium area patches decreases similarly to the overall decrease of NP for all the images (Fig. 5c). The vector layer preserves almost all the patches. The decrease with the two GEOBIA methods is much larger in the scaled raster method, because the medium patches are the first to be aggregated with the other larger ones, because of the decrease in the digital number variation in the image. The hierarchical GEOBIA aggregation of the medium and large patches is not dependent on the size but rather on the heterogeneity. Nevertheless the hierarchical GEOBIA aggregated the medium patches much earlier than the large patches, probably because of the similarity of the adjacent ones.

Coniferous forests were chosen as an example of the large patches (Fig. 5d). The aggregation effect was similar to the medium size patches in all three methods. The only change observed was that the GEOBIA raster aggregated the large patches on the basis of similarity at the beginning of the procedure. During the next levels the aggregation was limited.

\section{Shape of the objects}

To analyse the behaviour of the objects' shapes three metrics were chosen: Total Edge (TE) to characterize the general character of the edges in the whole landscape; Mean Patch Edge (MPE); and Mean Shape Index (MSI), intended to explore the shape of individual objects.

The Total Edge of objects presented in Figure 6a, is calculated by summing the perimeters of all the patches in the landscape. The vector scaling is the most generalizing approach, but the TE decreases relatively slowly because the number of patches remains high. Conversely, the GEOBIA raster method produces a relatively low number of large patches; although all the objects have a complicated shape, the TE does not represent these characteristics. The hierarchical GEOBIA produces a lower number of patches of complicated shape, and was expected to have higher values than TE, but the result is dependent on the number of patches.

The tendencies are well illustrated by Mean Patch Edge (MPE) metrics, calculated by normalizing the TE by the number of patches $(\mathrm{MPE}=\mathrm{TE} / \mathrm{NP})$, as presented in Figure $6 \mathrm{~b}$. There is a visible tendency by the GEOBIA raster to produce large complicated patches, a stable increase in complexity resulting from hierarchical GEOBIA, and smoothing of the results by generalization of the vector layer. The TE and MPE metrics are not the best methods for assessing the level of complexity in the whole landscape between the methods, but the problem of the influ- ence of the method on the shape metrics is well illustrated in this example. The results also explain why the influence of the scaling method on the pattern characteristics is often not recognized by users.

Much more informative representation of the shape characteristics normalized by areas of shape is provided by the Mean Shape Index (MSI) (Fig. 6c). The index is calculated with the following equation:

$$
M S I=\frac{\sum_{i=1}^{m} \sum_{j-1}^{n}\left(\frac{p_{i j}}{2 \sqrt{\pi \circ a_{i j}}}\right)}{N}
$$

where:

$$
\begin{aligned}
& a_{j}-\text { area }\left(\mathrm{m}^{2}\right) \text { of patch } \mathrm{ij} \\
& p_{j}-\text { perimeter }(\mathrm{m}) \text { of patch } \mathrm{ij}
\end{aligned}
$$

The index equals the sum of the patch perimeter $(\mathrm{m})$ divided by the square root of patch area $\left(\mathrm{m}^{2}\right)$ for each patch in the landscape, adjusted by a constant for a circular standard (developed for vector representation) and normalized by the number of patches (NP). The range of values is: $\mathrm{MSI} \geq 1$, without limit. The MSI equals one when all the patches in the landscape are circular, and increases when they become more irregular.

The MSI demonstrates the influence of the method on the results of the patch shape assessment quite well. The hierarchical GEOBIA is the only method which gives a stable increase trend of the MSI. It is caused by the nearly equal aggregation of the three patch sizes, which are merged with other neighbouring patches. The complexity of patches increases because the larger patches' borders preserve the shape of the borders of the patches on the lower level of scale. The aggregated patches become less circular, and more complicated, because of the proximity of other classes which are not aggregated with them. The other methods tend to reduce the shape complexity. The GEOBIA raster initially behaves similarly to the hierarchical GEOBIA. At a certain level of the raster image generalization (30 to 60) the small and similar patches are eliminated faster, and in consequence the resulting shapes become more circular. The complexity of the borders is also reduced, because the subsequent classifications are performed on aggregated raster images with pixels of increased dimensions. The vector aggregation smoothes the shape best of all methods; the overall trend is towards a round shape. 

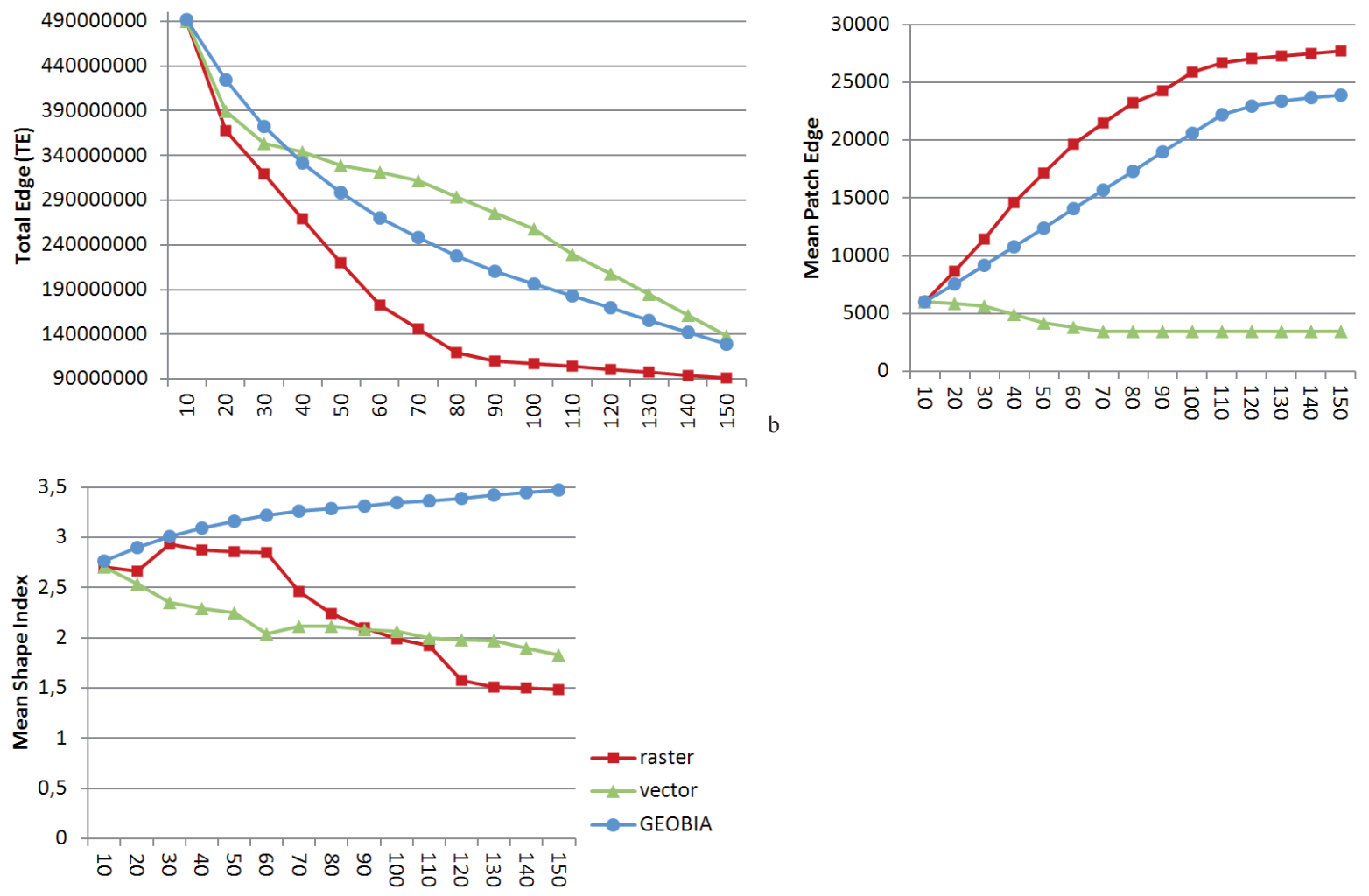

Figure 6. The perimeter (Total Edge) (a), Mean Patch Edge (b) Mean Shape Index (c) and of the objects in the layers scaled with the three methods: GEOBIA hierarchical scaling, GEOBIA raster and vector scaling

\section{Discussion and conclusions}

The results demonstrate the applicability of the three analysed methods of land cover layer scaling, which can be summarized for each method as follows.

The hierarchical GEOBIA preserves high comparability of the image objects between the scaled images. The small objects are not lost if they are distinguishable from the neighbourhood. The results bear strong relation to the truth on the ground, represented by the remote sensing scene. The overall shape of objects is not changed, and the complexity of the objects' border is only slightly smoothed. The scaled objects are applicable for characterizing landscape diversity, patch shape and edge complexity. Also, because of the comparability of results, the method allows patch spatial configuration analysis of neighborhood, core areas, and connectivity. The hierarchical GEOBIA is applicable whenever preservation with high accuracy is needed. The main limitation of the method is the low level of visual generalization of results (particularly borders), which can pose the problem of visualization of such a complex landscape at the lower scales.

The GEOBIA based on a scaled raster preserves the original shape of the objects. It effectively removes the unimportant similar patches. The method is useful for si- multaneous aggregation of objects' shape and attributes. Though the scaling process was applied, the shape of the objects can still be assessed. The method is also useful when landscape configuration analysis is needed, because it is usually performed on the generalized patches, and the individual small patches do not play a significant role. The highly accurate assessment of the neighborhood, core areas and connectivity is to be expected, e.g. forests contrasted with the arable land matrix. The method is not recommended for any analysis which assesses landscape diversity. Because of the removal of details, however, its results are suitable for visualization of generalized layers and also for generalization of class attributes.

The vector scaling method was applied without giving any weight to particular objects. Thus the small objects of important classes were removed. In its present version the method is not recommended for assessing diversity. Nevertheless after application of class weights the method may be applicable, because it preserves the original objects or they are merged with other neighbouring patches. The generalization of the shape leads to loss of the geometrical fidelity of the land cover patches, and thus the method is not recommended for assessing the complexity of landscape objects shape. Elements of the landscape configuration analysis can be assessed, but only when the level of 
layer generalization is known. The method is most suitable when schematic visualization is needed, facilitating understanding of the results.

As shown above, no one method is much better than the others or fit for every purpose. The results suggest that attention is needed when research methodology is developed which includes scaling categorical data. The adopted approach to data generalization strongly affects the results of research based on the calculated metrics.

The above results and methodological issues suggest further study. The methodology was examined for only one range of scales, types of land cover, without aggregation of patch attributes. The supposition is that it will also be suitable for other levels of landscape organization, e.g. for examining the difference between methods analysing the sub-objects of forest types e.g. trees or forest species, and super-objects, e.g. forested areas. This suggestion should be tested in further study. Also, such comparisons should look for different types of patch complexity and a range of spectral characteristics.

The other challenging issue is comparison of the classification results, which should be done in a more formal way when this is possible. Doing it on the basis of visual assessment of similarity depends on the operator and is therefore subjective. Ways of quantifying critical constraints of the methods need to be developed. The most important constraint relates to the level of scale of the GEOBIA segmentation results and the resolution of the base raster in relation to the heterogeneity of the patches. Also, quantification of the classification results assessment and of the dependence between the levels in the GEOBIA hierarchy of scales are frequently mentioned methodological challenges (e.g. Hay \& Castilla 2006).

The results of this study also prove that the Corine Land Cover layers are not universal to every application. Nevertheless the literature contains many examples of the characterization of landscape structure on that basis. The limitations of the vector scaling method described above suggest that CLC layers should not be used for calculation of landscape diversity to assess the shape of objects. The connectivity and core areas may give good results with this method, however.

\section{References}

Allen T. F. H. \& Starr, T. B., 1982, Hierarchy Perspective for Ecological Complexity, University of Chicago Press, Chicago.

Baatz M. \& Schaepe A., 2000, Multiresolution segmentation: an optimization approach for high quality multi-scale image segmentation, [in:] J. Strobl, T. Blaschke (eds.), Angewandte Geographische Informationsverar- beitung, Vol. XII ed., Wichmann, Heidelberg, Germany: $12-23$.

Bossard M., Feranec J. \& Otahel J., 2000, Technical report $N^{\circ} 40$, CORINE land cover technical guide, Addendum 2000, European Environment Agency.

Brown N., Gerard F. \& Fuller R., 2002, Mapping of land use classes within the CORINE land cover map of Great Britain, Cartographic Journal 39: 5-14.

Drăguţ L., Tiede, D. \& Levick S. R., 2010, ESP: a tool to estimate scale parameter for multiresoluti(on image segmentation of remotely sensed data, International Journal of Geographical Information Science 24 (6): 859-871.

EBONE, 2011, EBONE European Biodiversity Observation Network, http://www.ebone.wur.nl/UK/

EEA, 2007, Technical report No 17/2007, CLC2006 technical guidelines, EEA (European Environment Agency), OPOCE (Office for Official Publications of the European Communities).

Haralick R. M., 1979, Statistical and Structural Approaches to Texture. Proceedings of the IEEE. 67 (5): 786-804.

Hay G. J. \& Castilla G. C., 2006, Object-Based Image Analysis: strengths, weaknesses, opportunities and threats (SWOT), Bridging Remote Sensing and GIS: International Symposium on Object-based Image Analysis. 4-5 July, Salzburg, Center for Geoinformatics, International Archives of the Photogrammetry, Remote Sensing and Spatial Information Science, Com VI, WG VI/4: 5 .

Hay G. J., 2002, Multiscale object-specific analysis: An integrated hierarchical approach for landscape ecology. Ph. D. Thesis, Department of Biological Sciences, University of Montreal.

Hay G. J., Dubé P., Bouchard A. \& Marceau D. J., 2002, A scale-space primer for exploring and quantifying complex landscapes, Ecological Modelling 153 (1-2): 27-49.

Heuvelink G. B. M., 1992, An interactive method for multidimensional simulation with nearest neighbor models, Proceedings of the 2nd CODATA Conference on Geomathematics and Geostatistics, Nancy, France: 51-57.

Hunter G. J. \& Goodchild M. F., 1997, Modeling the Uncertainty of Slope Gradient and Aspect Estimates in Spatial Databases, Geographical Analysis 29 (1): 3549.

Kozioł K., 2012, Znaczenie punktów stałych obiektu w procesie upraszczania [The Importance of fixed points in the simplification proces], Archiwum Fotogrametrii, Kartografii i Teledetekcji 23: 169-177.

Kurczyński Z., 2006, Lotnicze i satelitarne obrazowanie Ziemi [Aerial and satellite Earth imaging], Oficyna Wydawnicza Politechniki Warszawskiej, Warszawa 2006: 388-394. 
Levin S. A., 1992, The problem of pattern and scale in ecology, Ecology 73: 1943-1967.

Lindeberg, T., 1994, Scale-space theory: A basic tool for analyzing structures at different scales, Journal of Applied Statistics 21 (2): 225-270.

Marceau D. J., 1999, The scale issue in the social and natural sciences, Canadian Journal of Remote Sensing 25 (4): 347-356.

Marceau D. J. \& Hay G. J., 1999, Remote sensing contributions to the scale issue, Canadian Journal of Remote Sensing 25 (4): 357-366.

Marceau D. J., Howarth P. J. \& Gratton D. J., 1994, Remote sensing and the measurement of geographical entities in a forested environment. Part 1: The scale and spatial aggregation problem, Remote Sensing of Environment 49 (2): 93-104.

McGarigal K., Landscape Pattern Metrics, [in:] J. Wiley \& Sons, Encyclopedia of Environmetrics, Ltd 2006.

MCPFE, 2011, Forest Europe, Ministerial Conference on the Protection of Forests in Europe, http://www.foresteurope.org/
SEBI, 2012, Streamlining European Biodiversity Indicators, Biodiversity Information System For Europe, http://biodiversity.europa.eu/topics/sebi-indicators/

Solon J., 2004, Ocena różnorodności krajobrazu na podstawie analizy struktury przestrzennej roślinności [Landscape diversity assesment based on landscape structure analysis], Działalność Nauk. 17: 110-112.

Tobler W., 1988, Resolution, Resampling, and All That, [in:] H. Mounsey, R. Tomlinson (eds.), Building Data Bases for Global Science, London, Taylor and Francis: 129-137.

Wang Z. \& Müller J. C., 1998, Line Generalization based on Analysis of Shape Characteristics, Cartography and Geographical Information Systems 25 (1): 3-15.

Wu J., Jelinski D. E., Luck M. \& Tueller P. T., 2000, Multiscale analysis of landscape heterogeneity: scale variance and pattern metrics, Geographic information science 6 (1): 1-19.

Wu J., 2004, Effects of changing scale on landscape pattern analysis: scaling relations, Landscape Ecology, Springer Netherlands: $125-138$. 
